\title{
The Alternative Universe
}

\author{
Luciano Rota \\ ${ }^{1}$ Sportmark 104, Almere $1355 \mathrm{KH}$, Netherland \\ Correspondence: Luciano Rota, Sportmark 104, Almere 1355 KH, Netherland. Tel: \\ 31-641-430-644/31-367-506-080. E-mail: lmrrota@live.nl
}

Received: March 26, 2012 Accepted: April 24, 2012 Online Published: July 20, 2012

doi:10.5539/apr.v4n3p123 URL: http://dx.doi.org/10.5539/apr.v4n3p123

\begin{abstract}
Starting from the several important experimental results on field of light propagations, obtained from 1865 till today, by indicating and describing a different natural source of waves, introducing new considerations on field of Theoretical Physics and suggesting a possible experiment to confirm the present hypothesis, this writing leads synthetically to conclude that electromagnetic waves are not, as normally understood, to be considered as classical waves and so, not connectable to the Doppler-shift. Consequently, from this angle, they offer a sustainable alternative interpretation of de red-shift of light coming from the most far from us celestial bodies.
\end{abstract}

Keywords: light propagation, waves, particles, Doppler-shift, redshift

\section{Introduction}

In classical physics the principle of "waves" is connected to the concept of rippling of the material by the material itself. Sound, movements of water which propagate across its surface or the vibrations that run along a wire.

What the three kinds of waves described above have in common is the fact that they need a material substance through which to propagate, be it in a gassy, liquid or solid state. The speed of these waves is, therefore, calculated in relation to the material substance in which they occur.

Electromagnetic waves, for several aspects, are not similar to the above-mentioned waves. There are some important differences, like:

1) They don't apparently need a field of any kind in which to propagate:

When we speak about "Doppler-shift" we implicitly speak of rippling of material substances trough the matter self. Any theorizing referring to the Doppler-shift must be connected to classical waves in the sense here above explained. Theoretical Physics indicates "magnetic fields" produced by the photons, as the possible material substance in which electromagnetic waves can occur. Not forgetting that photons have a mass 0 , we have logically and concretely to accept that a magnetic field needs energy to be produced. The question is: how many units of energy can produce "material" particles of mass $0(\mathrm{~m}=0)$ ? On ground of classic mechanic, as:

$$
E=1 / 20 C^{2}=0 \text {. }
$$

Means that a mass 0 cannot produce any kind of energy.

So that we remain with the following open question: if magnetic fields can be influenced by gravity, we have to understand that they must have a material consistence and consequently a non-zero mass, which represent a contradiction regarding the Special Relativity. When we consider photons and magnetic fields as massless, we cannot explain that gravity can have any influence on them.

2) Electromagnetic emanation consists in waves and particles as well.

The structure of magnetic waves on field of research can never be considered as a synthetic phenomenological context. Research can just be made on particles or, separately, on waves, treating the two parts of the same energy emanation as two different phenomena.

Differently, classical waves can be contained in a single context: there is a matter and rippling of the matter self.

3) Regarding the Doppler Effect, there isn't any difference when the source is moving from the observer or vice versa (or a difference which has been proved impossible to identify). 
Relatively to classical waves, we record two different kinds of shift regarding the mentioned two cases. About this question we will analytically see further on.

\section{Method}

We integrate those data in a single context, in order to concretely obtain an image of what the structure and the nature of magnetic waves concretely could be:

The most relevant data which we can make use of today is the knowledge of the fact that the particles making up matter contain a vibratory motion. As matter of facts it will be possible to consider the vibrations of an electron as a quick rotation around its nucleus: when we observe this phenomenon in a two-dimensional section, we could assume it as a quick vibration. However, it is also well-known that the speed these vibrations (or rotations) are directly proportional to the degree of heat of the matter in a relation that in rough synthesis we may define thus: the hotter the matter the faster its particles vibrate, the higher the frequencies it emanates.

Now let's imagine that, due to a magnetic reaction, these particles are literally fired into space in the form of continuous jets, at the original constant speed - in relation to the source - of approx. 300 thousand Km/s. Not forgetting that the particles in question have a vibratory movement, the result that we would obtain would be that of rippling fluxes, or better of particle waves, whose frequency would vary in relation to the degree of heating of the source emitting them. This looks like:



Figure 1.

A vibrating electron. It is already well known that each electron sends a photon. Let's imagine that those small particles together have been shot in a continuing flux from vibrating electrons. Then we see something like this:

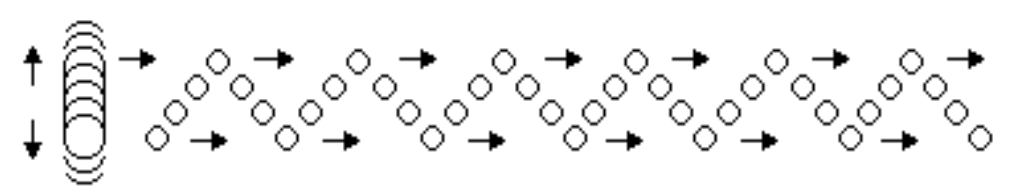

Figure 2.

It must be understood that, however each small particle follows a straight line; all parts together give the flux a waving motion.

Heated matter is never heated uniformly: usually the nucleus is the part most heated. The temperature gradually decreases towards the external parts of the matter. Making a relation between thermic degree and speed of the particles' vibration, we would logically find that the highest frequencies would be emanated from the hottest layers while the lowest from the coldest.

That means, the heater the matter, the faster the electrons vibrate, the higher the frequency of the waving flux, so that the distance between two wave tops become smaller:



Figure 3.

Coming back to the points we mentioned at the beginning of this writing we can see that this kind of structure offers us the following conclusions: 
1) These "waves" do not need any material substance in which to propagate. Since they originate from the source that produces them, they can propagate even through vacuum and carry on by inertial force. In the absence of gravity and agents of attrition, we could suppose that the speed originally imparted and so the frequency of the waves remains unvaried (constant) to the infinite.

2) From this point of view we can see how the - inside the official science -unexplainable duality between waves and particles can be totally and concretely explained: looking at this structure we can easily conclude that we are dealing with waves and with particles emanation as well. In facts, are the particles making up a wavy flux. This would be the only way to concretely connect waves and particles emission in asynthetic phenomenological context.

3) Regarding the Doppler Effect, in this hypothesis the behavior of the waves in relation to the frequency variations ascribable to the relative motion is perfectly coherent with the premise. In the first place, we must make it clear that, in the present hypothesis, the variation recorded in relation to the source's movements with respect to the observer, or vice versa, are not a consequence of the Doppler effect: in the sense that they do not represent a superimposition of frequencies, but a variation, caused by the relative decrease in speed of the flux between source and observer.

\section{Results}

Relatively to the point 3 , which represents one of the mentioned differences between classical waves and electromagnetic waves, we can obtain de following results:

a) Speed of vibration $=f / s$. Speed of the flux $=\mathbf{V u}$. The observer is moving from the source with velocity V. The distance between two wave-tops is:

$$
d=\frac{1}{f} \text { Of } V u
$$

or

$$
d=\frac{V u}{f}
$$

How many wave-tops $(\mathbf{w})$ receive the observer $(\mathbf{O})$ ?

$$
\begin{gathered}
O=a_{o}+V_{t} \\
W_{1}=b_{0}+V_{u} t \\
W_{1(0)}=O_{(0)} \Rightarrow b_{0}=a \\
\left.W_{2} \Delta t\right)=O(\Delta t)=>b_{0}-d+V_{u} \Delta t=a_{0}+V \Delta t \\
-d=V \Delta t-V_{u} \Delta t=\left(V-V_{u}\right) \Delta t \Rightarrow \Delta t=\frac{-d}{V-V u}=\frac{d}{V u-V} \\
\Delta t=\frac{V u}{(V u-V) f} \\
f_{0}=\frac{1}{\Delta t}=\frac{(V u-V) f}{V u}
\end{gathered}
$$

b) The source is moving from the observer with velocity $V$ :

$$
\begin{gathered}
\mathrm{d}^{\prime}=\frac{\mathrm{Vu}-\mathrm{V}}{\mathrm{f}}+\frac{\mathrm{V}}{\mathrm{f}} \\
\mathrm{d}^{\prime}=\frac{\mathrm{Vu}-\mathrm{V}+\mathrm{V}}{\mathrm{f}}=\frac{\mathrm{Vu}}{\mathrm{f}} \\
\mathrm{W}^{\prime}{ }_{1}=\mathrm{a}_{0}+(\mathrm{Vu}-\mathrm{V}) \mathrm{t} \\
\mathrm{W}_{2}{ }_{2}=\mathrm{a}_{0}-\mathrm{d}^{\prime}+\left(\mathrm{V}_{\mathrm{u}}-\mathrm{V}\right) \mathrm{t}
\end{gathered}
$$




$$
\begin{gathered}
\mathrm{O}^{\prime}=\emptyset \\
\left.W_{l_{(0)}}=O^{\prime}{ }_{0}=>a_{0}=\emptyset{ }^{\prime} \rightarrow W_{1} \Delta t\right)=O^{\prime}(\Delta t)=> \\
\mathrm{a}_{0}-\mathrm{d}^{\prime}+\left(\mathrm{V}_{\mathrm{u}}-\mathrm{V}\right) \Delta t=\emptyset \rightarrow d^{\prime}+(V u-V) \Delta t=\emptyset \\
\Delta t(V u-V)=d^{\prime} \\
\Delta t=\frac{d^{\prime}}{(V u-V)} \\
f^{\prime}{ }_{0}=\frac{1}{\Delta t}=\frac{(V u-V)}{d^{\prime}}=>f^{\prime}{ }_{0}=\frac{(\boldsymbol{V u}-\boldsymbol{V}) \boldsymbol{f}}{\boldsymbol{V u}}
\end{gathered}
$$

As we can see in both of cases there is no difference. In $v$ (relative separation speed) we find the decrease of frequencies originally emitted, as we logically expect that f' contains a decrease of the original speed of light due to the relative motion between source and observer.

To make this point clear, we have to suppose that when a source is moving with respect to an observer the latter records an increase or decrease in frequency due to the fact that the speed of the flux in relation to the observer changes so that, during the same measure of time, the latter receives a lower or higher number of waves -tops than if the source were stationary. The same lower or higher number recorded when the observer moves with respect to a stationary source.

\section{Discussion}

When we compare light-waves to the above described kind of waves we will discover in the first place, as this hypotheses is basically part of an "emission theory" and specifically "original source" (the velocity of light depends on the motion of the source), will be confirmed by the following experimental results :

1) Maxwell:

In 1865 has been proved that electromagnetic phenomena propagate trough space in the form of waves. Maxwell previewed that the speed of these waves will remain constant. We have to consider that the constancy of this speed results just when it is measured inside a limited coordinate-system. Light-speed has been measured the first time by the Danish astronomer Rømer, using one of the satellites of Jupiter on the distance that separate earth from Jupiter; and later corrected by Fizeauinterpolating frequency and wavelength. So that we have to consider that the speed of light has originally beenmeasured between a source and an observer. To assume the speed of light as a universal constant speed, we should relate a limited, measured speed between source and observer (c) to an unlimited (as far as we know) coordinate-system $(\infty)$. So that we have to conclude,sincec/ $\infty=0$. that any object, in absence of a parameter, moving in an unlimited space, has to be considered as static.

\section{2) Michelson-Morley:}

This result, obtained in 1887 , was originally directed to confirm the existence of a material substance (ether) in which light-waves could propagate. As well-known the result of this experiment leaded to exclude any form of etheric substance present in space. The premise of this experiment was grounded on the speed of earth around the sun. It was expected that the speed of light measured in the direction favorable to the earth's rotation would record a difference of $4 / 10$ of point on the spectrum, than when measured in a neutral direction, as consequence of the Doppler-shift. As this result did not bear out the expectation (the four tenth of a point shift on the spectrum turned out to be less than a twentieth. Practically no difference at all), the interpretation of this result was not only that to exclude the existence of ether, but also to suggest a constancy of light-speed independently from the moving of the source trough the space. This experimental result can give us an ulterior interpretation: as this experiment is carried out in absence of a relative motion between source and observer, we can also conclude there is no question of Doppler-shift when a source is moving through space in absence of relative motion with regard to its observer. So that,from this angle, in conformity with classic mechanic, also the speed of light, like any other measurable speed between two object can record an increase or decrease when calculated in relative motion.

De following experiments, since they are carried out on ground of de movements of sources trough space, they did not come out to ashift on the spectrum.

3) Fizeau convection coefficient 


\section{4) Aberration \\ 5) Kennedy-Thorndike \\ 6) Moving sources and Mirrors}

Experimental results like Michelson-Morley using sun light and De Sitter Spectroscopic Binaries which disagree with the "original source" theory, are starting from the ground of traditional waves, so that they make use of the Doppler calculations which leads to the conclusion that speed of light remains constant when measured in relative motion. From the premises of the here described waves, we can consider the two above mentioned experiments as a confirmation also for the present hypotheses, as we expect that the difference between $\boldsymbol{f}$ and $\boldsymbol{f}$ ' calculated by the Doppler, exactly the same is as that calculated on ground of an increase or decrease of the relative motion, when the source is moving with respect to the observer. Starting from the premise of a Doppler effect, on contrary, we have to detract the increase or decrease of frequencies as consequences of the shift, concluding that the speed of light remains constant even when a source is moving to or from an observer.

Just to resume, when we could accept that increase or decrease of original emitted frequencies are consequences of increase of decrease of the relative speed of magnetic waves between source and observer, and not of the Doppler shift, we will find that the this hypotheses agree with all results on the field of light propagation experiments.

The discovery made by Edwin Hubble, published in 1929, concerning the red-shift of the most far celestial bodies, led to the amazing conclusion that the farther away a celestial body is, the faster it moves away from us. The interpretative key to this phenomenon is, once again, given by Doppler.

Starting from the premise offered by the kind of waves we are dealing with, the interpretation of the red-shift phenomenon would be in conformity to the natural laws that we know.

If we imagine the particle fluxes - as they are presented in this hypothesis - emitted in the various frequencies of the spectrum of the most distant celestial bodies, it would be easy for us to suppose that, during the great distance they cover to reach us, they gradually exhaust the kinetic thrust originally obtained. And this could happen because of the fact that said fluxes cross innumerable gravitational fields which break the initial thrust. In terms of logical consequence, the result would be that the farther the energy sources under consideration are, the more the fluxes reaching us appear to slow down. This would be perceived under spectroscopic analysis, as a shift of colors towards red. As it is explained before, once again, it would not be the Doppler Effect, in the sense that we know, but a real slowing-down of the original speed of the fluxes and its consequence, which has been registered as a decrease of the frequencies globally emitted.

The relation distance/red-shift would give us, in these terms, a logical and convincing explanation.

It has been suggested that celestial bodies, when bypass a certain big distance from each other can invert the attraction thrust of gravity into expulsion force, in order to may obtain the trust to constantly accelerate from an hypothetical nucleus of an original "big-bang" Before accepting this suggestion as a real possibility, we have to explain the following discovery made inside our system:

In September 1998 Dr. Anderson of de N.A.S.A. Department of Pasadena declared an unexplainable discover concerning the Pioneer 10 and 12 traveling at that moment trough the frontier of our system. These two space objects seem to increase their speed, without any apparent reason.

A reason can be found when we could start from the ground of the kind of waves here explained: we know the two objects are moving from us at a certain constant speed, so that the radio signals we receive are charged of a decrease in frequencies due to - we presume - the Doppler-shift. When the decrease in frequency is bigger than the Doppler-shift of the speed of moving away must be, and we consider the speed of radio waves as a constant factor, than we must conclude that the speed of the two Pioneers increases as more as they increase their distance from us.

From the other point of view we can imagine that the flux of radio waves coming from the Pioneers decrease their speed due to the increasing distance from us and the gravity fields present in our system, so that the bigger is de distance from us the slower the fluxes of radio-waves reach us, the wider de distance between two wave-tops. From this angle is evident, there isn't any "strange power" which causes a real constant increase of speed. This conclusion is just the consequence of the fact we, most probably, continue to connect the Doppler-effect to phenomena that have nothing to deal whit it. 


\section{About Doppler-effect and Redshift}

\section{a) Doppler Effect}

Just to resume, the most relevant differences between classical waves and electromagnetic waves are:

1. The speed of classical waves must be calculated in relation of the matter in which they occur. The speed of electromagnetic waves must be calculated with regard to the source that emits them.

2. Treating of classical waves, we have to consider that an objective variation of the wavelength can be registered when a source is moving through a matter (Figure 4), but it remains constant when an observer is moving with regard to a stationary source (Figure 5)

Speaking of Electromagnetic waves we should assume that in both cases there is no difference between wavelength emitted and wave length observed, but just a difference of frequency that the observer records due to the relative motion.

Let us take a look at de following figures. Classical waves, first case: the source is moving to an observer with velocity $v$.

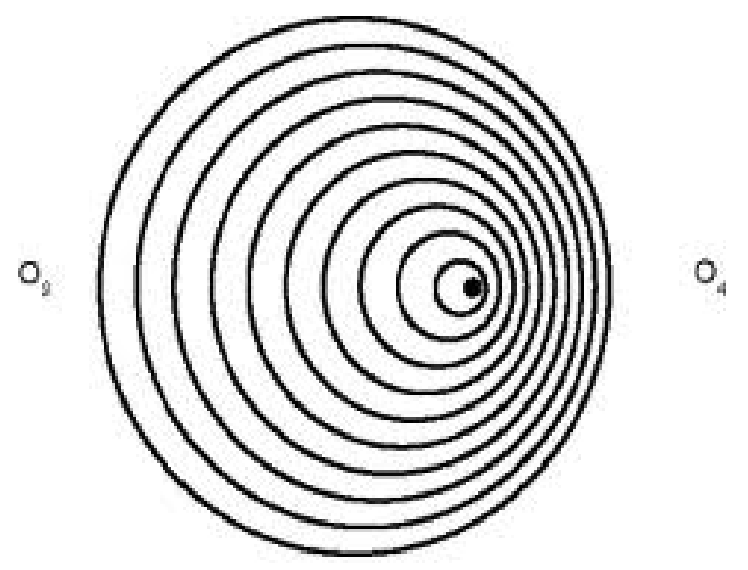

Figure 4. Velocity of source relative to observers is to the right

As we can see, the movements of the source trough the matter produce a real objective increasing or decreasing of the wavelength, so that the observer receives an increased or decreased frequency.

Second case: an observer is moving to or from a stationary source. The wavelength originally produced will remain constant, but the observer receives a different frequency due to the relative motion (Figure 5). In facts, we have to assume that in this case the differences between $f_{\text {emit }}$ and $f_{\text {obsv }}$ are not depending on a real objective variation of wavelength, but subjectively recorded by an observer due to the relative motion between the latter and the source.

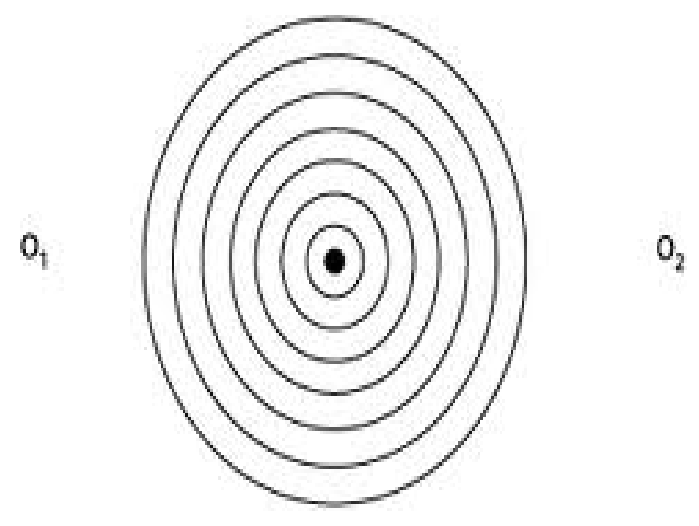

Velocity of source to observers $=0$

Figure 5. Observers move to and from source 
Dealing with electromagnetic waves, when an energy source is moving through space we have to suppose that all frequencies emitted would maintain the same wavelength in any case, being it in motion or being it stationary. What changes, is the frequency the observer receives just when a relative motion between the latter and the source exists. (Take a look at Figure 5 and imagine now the source is moving into space with regard to the observers).

\section{b) Red-shift}

If we start from the premise of a possible slowdown of light fluxes, in conformity to what above has been explained, we have to consider that the wavelength emitted the same is as the wavelength observed:

$$
(\lambda \text { emit }=\lambda \text { obsv })
$$

The measure that in this case has been perceived as a decreasing of wavelength is in fact that of a decreasing of the frequency the observer records due to the slowing down of the light fluxes:

$$
\left(f_{\text {emit }}>f_{\text {obsv }}\right)
$$

Let us take as an example the quasar known as 3C-273:

The hydrogen Balmer-alpha line of this body has been calculated in a wavelength of ( $\lambda$ emit) $760 \mathrm{~nm}$.

The wavelength observed ( $\lambda \mathrm{obsv}$ ) in $656 \mathrm{~nm}$.

The calculation of the redshift $(\mathrm{z})$ based on the difference of wavelength is therefor:

$$
z=\frac{\lambda_{\text {obsv }}-\lambda_{\text {emit }}}{\lambda_{\text {emit }}}=0,1386
$$

As explained before, in this case we have to consider the measure of $\lambda$ as aconstant of the emission. Whatwe are observing is not a difference of an observed wavelength but a decreasing of frequencydue to the slowdown of the light flux. The right way to calculate the red-shift $(\mathrm{z})$ based on frequency will be therefore:

$$
\mathrm{Z}=\frac{\lambda_{\text {obsv }}-\lambda_{\text {emit }}}{\lambda_{\text {fobsv }}}=0,1386
$$

Differently, the calculation of the redshift of this body based on frequency has been calculated in $\mathrm{z}=0,1585$ and needs a relativity correction in order to become to a correct result.

From this alternative way, we also cancalculate the value of the slowdown $(V)$ of the light flux and consequently that of the observed light speed $\left(C_{o b s v}\right)$ :

$$
V=C Z=(300,000)(0,1386)=41,400 \mathrm{KM} / \mathrm{s}
$$

And:

$C_{\text {obsv }}=C-C Z=(300,000)-(41.400)=258,600 \mathrm{Km} / \mathrm{s}$

So that:

$$
f_{o b s v}=\frac{(c-v) f_{\text {emit }}}{C}=656
$$

Through this way, as we can clearly see, we obtain a correct result without making use of a relativity correction.

If we should succeed to measure the speed of light coming from this or a similar far body, we will able to definitively confirm the present hypothesis.

\section{Conclusion}

All the data, calculations and suggestions included in the present writing can concretely and correctly lead to the conclusion that theoretical physics has probably started from a misunderstood dynamics of electromagnetic waves. When we could accept and prove that electromagnetic waves can be originated by kinetic thrust produced on material particles with mass and propagate through the space by inertial force, we can start to reconsider the origins of the universe grounded on its expansion. 


\section{References}

Anderson, J. D., Campbell, J. K., \& Nieto, M. M. (2006). The Energy Transfer Process in Planetary Flybys. New Astronomy, 12(5), 383. arXiv:astro-ph/0608087. http://dx.doi.org/10.1016/j.newast.2006.11.004

Anderson, J. D., Campbell, J. K., Ekelund, J. E., Ellis, J., \& Jordan, J. F. (2008). Anomalous Orbital-Energy Changes Observed during Spacecraft Flybys of Earth. Phys. Rev. Lett., 100(91102), 091102. http://dx.doi.org/10.1103/PhysRevLett.100.091102

NASA Baffled by Unexplained Force Acting on Space Probes. (2008). Retrieved from http://www.space.com 